\title{
EDITORIAL
}

\section{Why a new journal, Diabesity- editorial perspectives}

\author{
Sijo Joseph Thandapilly *
}

\begin{abstract}
I am very pleased to write an inaugural editorial for the new journal, Diabesity as the editor-in-charge. Diabesity is a unique peer-reviewed open access interdisciplinary journal dedicated to publishing high-quality articles in the areas of physiology, pathology and therapeutic aspects of diabetes, obesity and their complications. Diabesity focuses on clinical and experimental pharmacology and therapeutics in any aspect of these metabolic ailments in various experimental and clinical settings. Current therapeutic agents for obesity and diabetes are inadequate as the number of cases is on the rise. Along with public health strategies, development of alternative therapeutic agents is anticipated. Therefore, extensive research is being conducted in this field and is drawing intense attention from researchers, practitioners and the general public. Here arises the significance of a journal like Diabesity which can serve as a platform for knowledge sharing and the promotion and dissemination of new scientific advancements to a wide spectrum of readers.
\end{abstract}

Keywords: Obesity, diabetes, diabesity, metabolic disorders

\section{Introduction}

I am very pleased to write an inaugural editorial for the new journal, Diabesity as the editor-incharge. Diabesity is a unique peer-reviewed open access interdisciplinary journal dedicated to publishing highquality articles in the areas of physiology, pathology and therapeutic aspects of diabetes, obesity and their complications. Accordingly, Diabesity focuses on clinical and experimental pharmacology and therapeutics in any aspect of these metabolic ailments in various experimental and clinical settings.

As we all know, obesity and diabetes epidemic poses a major health-care challenge world-wide. ${ }^{1,2}$ An unhealthy lifestyle (high caloric diets and lack of physical activity) accelerates this epidemic, when superimposed with genetic susceptibility. 3,4 Current therapeutic agents for obesity and diabetes are inadequate as the number of cases is on the rise. Along with public health strategies, development of alternative therapeutic agents is anticipated. Therefore, extensive research is being conducted in this field and is drawing intense attention from researchers, practitioners and the general public. Here arises the significance of a journal like Diabesity which can serve as a platform for knowledge sharing and the promotion and dissemination of new scientific advancements to a wide spectrum of readers.

\section{Diabesity}

The term 'diabesity' is relatively new; and first emerged after the recent establishment of a strong link between obesity and diabetes substantiated through clinical evidence. As evident from recent clinical data, proliferation in the prevalence of obesity is closely paralleled by the increase in the prevalence of diabetes. These ailments exist as two sides of the same coin and the clinical and research community are trying to elucidate this link. The driving forces linking diabetes and obesity remain to be explained due in part to the fact that environmental, genetic, life style and behavioral factors are involved in the pathogenesis of these disease states. Therefore we believe there is room for a dedicated interdisciplinary journal in the area of diabesity despite the fact that there are many reputable journals which already exist in the areas of diabetes and obesity.

\section{Scope of Diabesity}

Diabesity publishes but is not limited to clinical and experimental pharmacology and therapeutics in any aspect of metabolic diseases, either in humans or animal models and in vitro systems. Diabesity also welcomes manuscripts on any aspect of physiological and pathological pancreatic islet function and intermediary metabolism, mechanisms of drug and hormone action, and biochemical and molecular aspects of normal and abnormal biological processes. In addition, articles encompassing clinical nutrition, adipogenesis and lipid metabolism, neuroscience, transgenic models, metabolic syndrome, nutrition behavior, pediatric obesity, and adipocyte cell biology are also of interest. 
Diabesity will accept original research articles, review articles, editorials, case reports and short communications on all the aspects of diabetes, obesity and metabolic syndrome. The journal has an online submission and editorial tracking system for a rapid and quality review process. The review process is carried out through the editorial board members or other experts from the field with the highest standards of professional ethics. The editorial board for this journal consists of prominent scientists around the world including countries such as Canada, the United States of America, Japan, Brazil, France, India and China.

\section{Concluding remarks}

Again, I welcome you to the inaugural issue of Diabesity, and I hope that researchers and clinicians exploring the diabesity enigma will turn to the journal Diabesity as a platform for their outstanding research work. I hope to hear from you, through your submissions, reviews, or letters to the editor to express your views and ideas.

\section{Conflict of interest}

Dr. Sijo Joseph Thandapilly is the editor-incharge for Diabesity.

\section{References}

1. Rodgers GP, Collins FS. The next generation of obesity research: no time to waste. JAMA. 2012; 19;308(11):1095-6. PubMed Full Text

2. Bailey CJ. The challenge of managing coexistent type 2 diabetes and obesity. BMJ. 2011; 13;342:d1996. PubMed Full Text

3. Hossain P, Kawar B, El Nihas M. Obesity and diabetes in the developing world - a growing challenge. $N$ Engl J Med. 2007; 356: 213-5. PubMed Full Text

4. Marti A, Moreno-Aliaga MJ, Hebebrand J, Martínez JA. Genes, lifestyles and obesity. Int J Obes Relat Metab Disord. 2004; 3:S29-36. PubMed 